\title{
Advanced Optical Signal Processing using Time Lens based Optical Fourier Transformation
}

Guan, Pengyu; Røge, Kasper Meldgaard; Lillieholm, Mads; Hu, Hao; Galili, Michael; Morioka, Toshio; Oxenløwe, Leif Katsuo

\section{Published in:}

Proceedings of Asia Communications and Photonics Conference 2016

Link to article, DOI:

10.1364/ACPC.2016.AS1H.1

Publication date:

2016

Document Version

Peer reviewed version

Link back to DTU Orbit

Citation (APA):

Guan, P., Røge, K. M., Lillieholm, M., Hu, H., Galili, M., Morioka, T., \& Oxenløwe, L. K. (2016). Advanced Optical Signal Processing using Time Lens based Optical Fourier Transformation. In Proceedings of Asia Communications and Photonics Conference 2016 [AS1H.1] Optical Society of America (OSA). https://doi.org/10.1364/ACPC.2016.AS1H.1

\section{General rights}

Copyright and moral rights for the publications made accessible in the public portal are retained by the authors and/or other copyright owners and it is a condition of accessing publications that users recognise and abide by the legal requirements associated with these rights.

- Users may download and print one copy of any publication from the public portal for the purpose of private study or research.

- You may not further distribute the material or use it for any profit-making activity or commercial gain

- You may freely distribute the URL identifying the publication in the public portal 


\title{
Advanced Optical Signal Processing using Time Lens based Optical Fourier Transformation
}

\author{
P. Guan, K. M. Røge, M. Lillieholm, H. Hu, M. Galili, T. Morioka, and L. K. Oxenløwe, \\ DTU Fotonik, Technical University of Denmark, Ørsteds Plads, 343, Kgs. Lyngby, 2800, Denmark \\ pengu@fotonik.dtu.dk
}

\begin{abstract}
An overview of recent progress on time lens based advanced optical signal processing is presented, with a special focus on all-optical ultrafast $640 \mathrm{Gbit} / \mathrm{s}$ all-channel serial-to-parallel conversion, and scalable WDM regeneration.

OCIS codes: (070.4340) Nonlinear optical signal processing; (060.4510) Optical communications
\end{abstract}

\section{Introduction}

The internet traffic demand has grown dramatically in the past decades. Recent bandwidth-hungry applications such as data sharing, transmission of ultra high definition video and cloud computing, is driving the need for higher capacities in signal transmission and signal processing [1]. Current electronic digital signal processing (DSP) technologies have been used to demonstrate transmissions with more than $1 \mathrm{Tbit} / \mathrm{s}$ capacity [2]. However, due to the bandwidth limitation of electronics, the number of required components and their combined power consumption scales unfavorably with the capacity. As an alternative, optical signal processing (OSP) that can offer very high bandwidth, and can avoid optical to electrical to optical (OEO) conversion, has the potential to realize energyefficient systems with very high capacity [3]. In general, OSP is considered suitable only for relatively simple functionalities. Whereas complex digital logic may turn out to be challenging to implement optically, format conversions or Fourier transformations are quite simple to implement optically. The time lens based optical Fourier transformation is an extremely attractive tool for such applications.

In this paper, we provide an overview of recent progress on advanced optical signal processing using time lens based optical Fourier transformation. We will outline time lens based ultrahigh-speed $640 \mathrm{Gbit} / \mathrm{s}$ all-channel serialto-parallel conversion, and scalable wavelength division multiplexing (WDM) regeneration technologies.

\section{Time lens based optical Fourier transformations}

Time lens based optical Fourier transformation is a very versatile tool which allows for a large range of optical communication applications [4]. It can transfer the temporal profile of an optical signal into the frequency domain and vice versa. Time lenses are based on the principle of the space-time duality of light, which states that a quadratic phase modulation of a temporal waveform is analogous to the action of a thin lens on a spatial beam, hence the expression "time lens" [5]. Similar to a spatial lens system, by combining a time lens with suitable dispersion media, four different OFTs can be realized as shown in Fig. 1. Fig. 1(a) shows a partial OFT with a K-D configuration that can realize frequency-to-time conversions. It is based on a quadratic phase-modulation stage $\left(\delta \phi=K t^{2} / 2\right)$ with chirp rate $K$, followed by a dispersive medium with $D=\beta_{2} L$ (where $\beta_{2}$ is the 2nd order dispersion and $L$ is the length), which satisfies the condition $K=1 / D$. Such an OFT has been used for distortion-less transmission [6] and WDM to optical time-division multiplexing (OTDM) conversion, achieving single channel $2.56 \mathrm{Tbit} / \mathrm{s}$ transmission over $300 \mathrm{~km}$ [7], and 16×10 Gbit/s WDM to a $160 \mathrm{Gbit} / \mathrm{s}$ OTDM conversion [8]. In contrast with Fig. 1(a), Fig. 1(b) shows a D-K configuration that can realize time-to-frequency conversions. Some representative applications include an ultra-fast optical oscilloscope with 220-fs resolution [9] and a $640 \mathrm{Gbit} / \mathrm{s}$ OTDM-to-WDM conversion [10]. Fig. 1(c) shows the traditional OFT configuration (a D-K-D configuration), as described by A. W. Lohmann in 1992 [11]. It can realize simultaneous frequency-to-time and time-to-frequency conversion. However, the dispersive

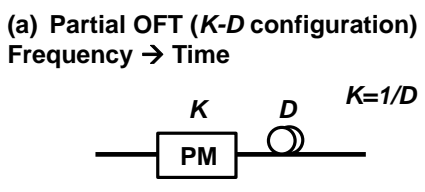

(c) Traditional OFT ( $D-K-D$ configuration) Frequency $\rightarrow$ Time, and Time $\rightarrow$ Frequency

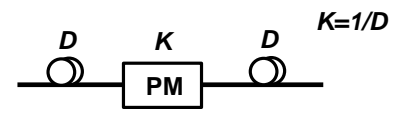

(b) Partial OFT ( $D-K$ configuration)

Time $\rightarrow$ Frequency

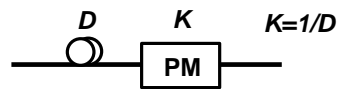

(d) Complete OFT ( $K-D-K$ configuration)

Frequency $\rightarrow$ Time, and Time $\rightarrow$ Frequency

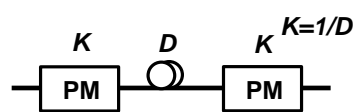

Fig. 1. Four different OFTs, (a) partial OFT with K-D configuration, (b) Partial OFT with D-K configuration, (c) traditional OFT with D-K-D configuration, (d) new complete OFT with K-D-K configuration. 
(a)

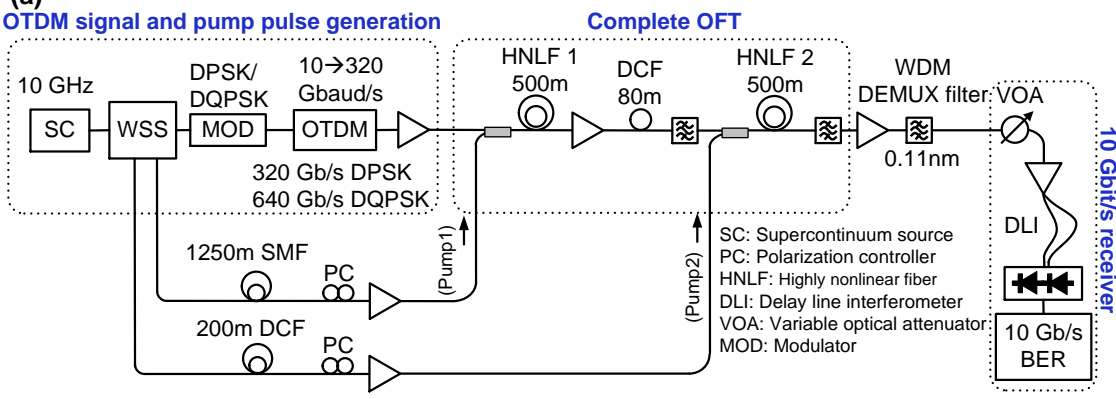

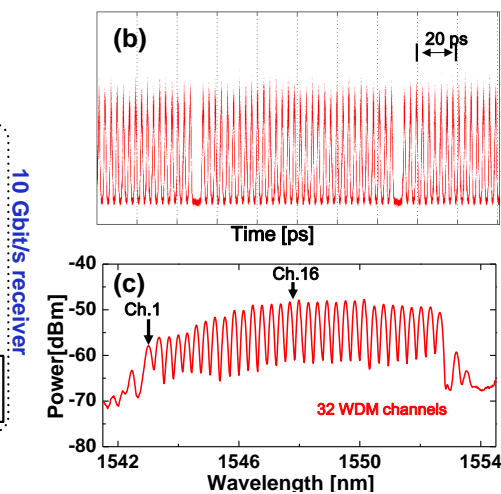

Fig. 2. (a) Experimental setup for all-channel OTDM to WDM conversion of a 640-Gbit/s-DQPSK signal, (b) 320 Gbaud OTDM signal waveform, (c) spectrum of the 32 obtained WDM channels.

elements before the phase modulation stage will generally broaden the input waveform beyond its time slot, causing large inter-channel crosstalk and OSNR degradation, hence limiting the application scope of this configuration. To overcome this challenge, we proposed a new time lens based complete OFT with a K-D-K configuration, cf. Fig. 1(d). This configuration pre-chirps the input signals at the first phase modulation stage, thus keeping the waveform within the time lens apertures, making this configuration more versatile for ultrahigh-speed optical signal processing applications. It has been used to achieve many advanced optical signal processing applications, e.g. real time all-optical (AO) $160 \mathrm{Gbit} / \mathrm{s}$ OFDM transmission [12], WDM to spectrally efficient Nyquist channel conversion [13], and AO $640 \mathrm{Gbit} / \mathrm{s}$ OFDM to Nyquist WDM conversion [14].

\section{Ultrahigh-speed $640 \mathrm{Gbit} / \mathrm{s}$ all-channel serial-to-parallel conversion}

The partial OFT with a D-K configuration has been used to demonstrate serial-to-parallel conversion [10]. However, such an OFT allows only a part of the serial channels to be converted to parallel channels. An all-channel serial-toparallel converter where all channels are simultaneously converted may become a very important functionality, since it enables the simultaneous processing of a large number of parallel channels (e.g. WDM data) in a single device. Recently, we have demonstrated a $640 \mathrm{Gbit} / \mathrm{s}$ ultrahigh-speed all-channel serial-to-parallel conversion using a complete OFT with a K-D-K configuration, which greatly reduces the inter-channel crosstalk and OSNR degradation [15]. In this demonstration, a high-speed 320-Gbaud serial data signal is generated by OTDM. The experimental setup is shown in Fig. 2(a). We generated a 1-ps Gaussian pulse train using a supercontinuum source and a programmable wavelength selective switch (WSS). The obtained pulse train is DPSK or DQPSK modulated using an IQ modulator. The 10-Gbaud modulated signal is then OTDM-multiplexed to 320-Gbaud, resulting in a 320-Gbit/s DPSK-OTDM signal or a 640-Gbit/s DQPSK-OTDM signal. Fig. 2(b) shows the obtained 320-Gbaud OTDM signal waveform. A $3.4 \mathrm{ps}$ guard interval is inserted between every 32 tributaries for the OFT. A complete OFT is used for the all-channel OTDM-to-WDM conversion. The quadratic phase modulation is implemented based on a four-wave mixing (FWM) process in a highly nonlinear fiber (HNLF) using linearly chirped rectangular pump pulses. The spectrum after the complete OFT is shown in Fig. 2(c), where $32 \mathrm{WDM}$ channels with $37.5 \mathrm{GHz}$ $(0.3 \mathrm{~nm})$ spacing can be observed. The skew between the channels is caused by the limited FWM bandwidth of the used HNLFs. This indicates nearly insignificant spectral channel overlap and negligible crosstalk, resulting in an allchannel serial-to-parallel conversion. Finally, the BER of each demultiplexed DPSK or DQPSK channel is measured in a pre-amplified receiver. All 32 tributaries of a 320-Gbit/s DPSK-OTDM signal were simultaneously converted to 32 DWDM channels, achieving a BER $<10^{-9}$ performance. An all-channel 640-Gbit/s DQPSK-OTDM to WDM conversion was also demonstrated with a BER performance below the standard FEC threshold $\left(2 \times 10^{-3}\right)$. This scheme has the potential to extend existing serial signal processing techniques to include parallel processing

\section{Scalable WDM regeneration}

All-optical regeneration of WDM signals could play an important role in future optical communication systems, and phase sensitive amplifiers (PSAs) have emerged as a promising all-optical way to regenerate phase-encoded signals [16]. However, it is very challenging to extend PSA schemes to WDM regeneration as it requires multiple pumps and the data signal carriers to be in-phase at the PSA input. Recently, we proposed a novel all-optical WDM regeneration scheme for DPSK signals based on OFT and phase sensitive amplification [17]. The principle of alloptical WDM regeneration of DPSK signals is shown in Fig. 3(a). A number of WDM DPSK channels (A) with channel spacing $\Delta \boldsymbol{v}$ are passed through a 1-bit delay interferometer (DI) and converted to OOK signals (B). A partial OFT with K-D configuration is employed for WDM to OTDM conversion. After the OFT, the WDM signal is thus converted to an incoherent OTDM OOK signal (C). XPM in a highly nonlinear fiber (HNLF) is employed to 

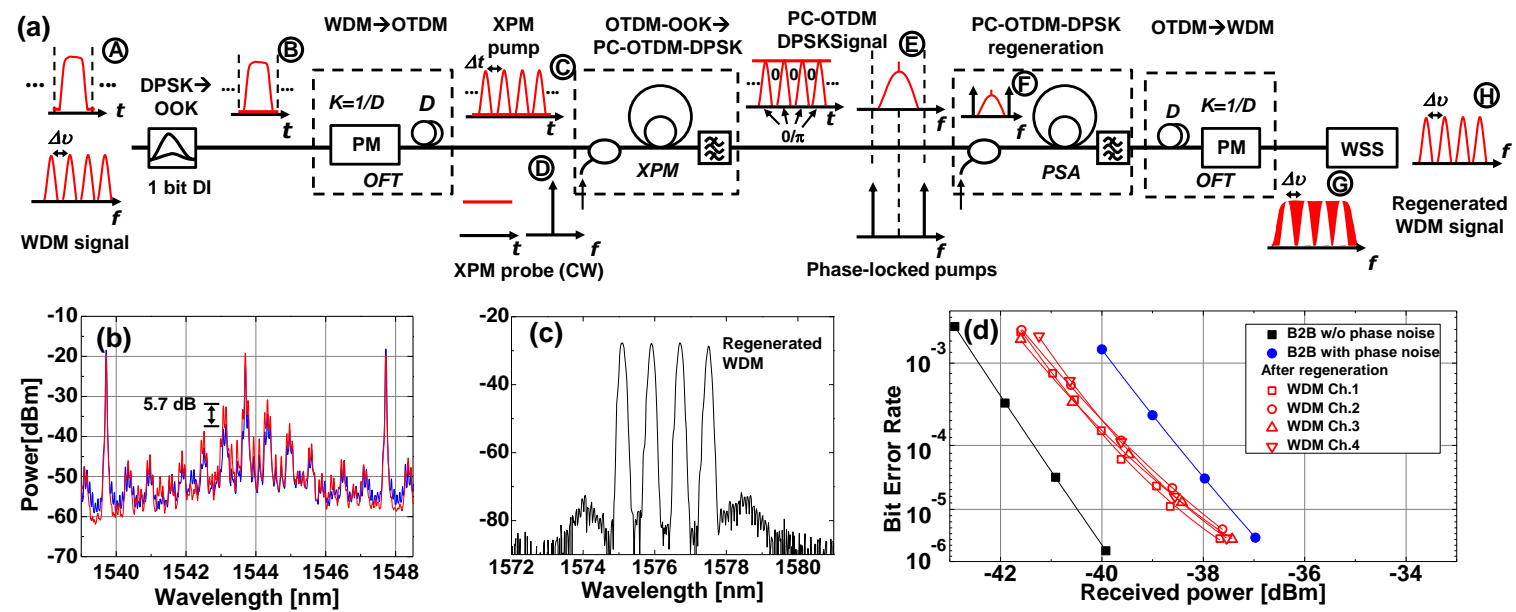

Fig. 3. (a) Principle of all-optical WDM regeneration of DPSK signals, (b) spectra at the PSA output for maximum (red) and minimum (blue) output signal power, (c) the regenerated WDM signal, (d) BER performance of the regenerated WDM channels.

transfer the data modulation from the OOK signal to the phase of a continuous wave (CW) carrier (D), resulting in a phase coherent OTDM (PC-OTDM DPSK) signal (E). After combination with two phase-locked pumps, the obtained PC-OTDM DPSK signal (F) is sent to a HNLF to achieve phase sensitive amplification for phase regeneration. The second OFT is then used to convert the regenerated PC-OTDM channels back to WDM channels (G). The unmodulated CW parts are finally removed by a WSS (H). The phase sensitive extinction ratio of the PSA is $5.7 \mathrm{~dB}$ as shown in Fig. 3(b). Fig. 3(c) shows the spectrum of the regenerated WDM channels. Fig. 3(d) shows the BER performance of 4 regenerated phase noise degraded DPSK WDM channels. The power penalty is $2.6 \mathrm{~dB}$ at $\mathrm{BER}=10^{-3}$, when phase noise is added. After WDM regeneration, the power penalty is reduced by $1.3 \mathrm{~dB}$ for all WDM channels. The system complexity of the proposed scheme does not scale with the number of WDM channels, making the scheme attractive for processing a large number of WDM channels.

\section{Conclusion and Acknowledgments}

We have reviewed recent progress in advanced all-optical processing using time lens based OFT. Time lens based OFT has the benefits of allowing for ultrafast, complex OSP, and may become a very important enabling technology for future optical communication systems.

The authors would like to acknowledge Danish Research Council: FTP project LENS-COM (ref. DFF-505400184), FTP project TOR (ref. no. 12-127224), DFF Sap. Aude Adv. Grant NANO-SPECs and OFS Denmark.

\section{References}

[1] A. E. Willner et al., "All-Optical Signal Processing," IEEE JLT., 32(4), pp. 660-680, (2014).

[2] X. Liu et al., "Digital Signal Processing Techniques Enabling Multi-Tb/s Superchannel Transmission: An Overview of Recent Advances in DSP-Enabled Superchannels"IEEE Signal Process. Mag., 31(2), 16-24, (2014).

[3] H. C. H. Mulvad et al., "Recent advances in ultra-high-speed optical signal processing," ECOC2012, paper Tu1A5, (2012).

[4] L.K.Oxenløwe et al, Ch. 8, Eds. B.J. Eggleton, S. Wabnitz, Springer, DOI 10.1007/978-3-319-14992-9 (2015).

[5] B. H. Kolner et al., "Temporal imaging with a time lens," Opt. Lett., 14(12), pp. 630-632, (1989).

[6] M. Nakazawa et al., "Ideal distortion-free transmission using optical Fourier transformation and Fourier transform-limited optical pulses", IEEE PTL., 16(4), pp. 1059-1061, (2004).

[7] P. Guan et al., "2.56 Tbit/s/ch Polarization-Multiplexed DQPSK Transmission over 300 km Using Time-Domain Optical Fourier Transformation," Optics Express 19(26), pp. B567-73, (2011)

[8] H. C. H. Mulvad et al., "DWDM-to-OTDM conversion by time-domain optical Fourier transformation," ECOC.2011, paper Mo.1.A.5, (2011).

[9] M. A. Foster et al., "Silicon-chip-based ultrafast optical oscilloscope," Nature, vol. 456, pp. 81-84, (2008).

[10] H. C. H. Mulvad et al., "Ultra-High-Speed Optical Serial-to-Parallel Data Conversion in a Silicon Nanowire," ECOC2011. Th13A2, (2011).

[11] A. W. Lohmann et al., "Temporal filtering with time lenses," Appl. Opt., 31( 29), pp. 6212-6219, (1992).

[12] P. Guan et al., "Real-Time All-Optical OFDM Transmission System Based on Time-Domain Optical Fourier Transformation." OFC 2014, paper W4F.1, (2014).

[13] P. Guan et al., "Conversion of a DWDM Signal to a Single Nyquist Channel based on a Complete Optical Fourier Transformation." ECOC2014, paper We.2.5.5, (2014).

[14] P. Guan et al., "All-Optical Ultra-High-Speed OFDM to Nyquist-WDM Conversion Based on Complete Optical Fourier Transformation," IEEE JLT., 34(2), pp. 626-632, (2016).

[15] P. Guan et al., "Ultra-high-speed All-channel Serial-to-parallel Conversion based on Complete Optical Fourier Transformation," OFC 2016, paper W3D.2, (2016).

[16] R. Slavík et al., "Practical issues and some lessons learned from realization of phase sensitive parametric regenerators," OFC2012, paper OW3C4, (2012),

[17] P. Guan et al., "All-optical WDM Regeneration of DPSK Signals using Optical Fourier Transformation and Phase Sensitive Amplification," ECOC2015, paper We3.6.4, (2015). 